Published in final edited form as:

Curr Hypertens Rep. 2013 April ; 15(2): 102-107. doi:10.1007/s11906-013-0330-y.

\title{
Aldosterone and the Risk of Hypertension
}

\author{
Vanessa Xanthakis, $\mathbf{P h D}^{1,3}$ and Ramachandran S. Vasan, $\mathbf{M D}^{1,2,3}$ \\ ${ }^{1}$ Sections of Preventive Medicine and Epidemiology and Cardiology, Boston University School of \\ Medicine, Boston, MA, USA \\ ${ }^{2}$ Department of Medicine, Boston University School of Medicine, Boston, MA, USA \\ ${ }^{3}$ Framingham Heart Study, Framingham, MA, USA
}

\begin{abstract}
Aldosterone, the key hormone in the mineralocorticoid pathway, plays a fundamental role in salt and water homeostasis, blood pressure regulation, and cardiovascular remodeling. Both genomic and non-genomic mechanisms influence aldosterone-induced renal sodium reabsorption. Furthermore, the mineralocorticoid receptor in non-epithelial tissues, including the heart and vascular smooth muscle cells, have recently been discovered. Thus, aldosterone likely has pleiotropic effects that contribute to the modulation of blood pressure. Among patients with hypertension in general, and among those with more severe or resistant hypertension in particular, a higher than expected prevalence of primary hyperaldosteronism is noted. Among individuals with resistant hypertension, aldosterone antagonists have also been shown to be effective in lowering blood pressure. Most significantly, recent community-based studies among nonhypertensive individuals in the general population have demonstrated that both a higher serum aldosterone concentrations and a higher aldosterone to renin ratio portend a greater risk of developing hypertension. The combination of the aforementioned observations underscores the importance of the mineralocorticoid pathway in the pathogenesis of hypertension.
\end{abstract}

\section{Keywords}

Aldosterone; Mineralocorticoid receptor; Aldosterone/renin ratio; ARR; Primary hyperaldosteronism; Natriuretic peptides; Salt sensitivity; Blood pressure; Hypertension; Cardiovascular disease; Risk

\section{Introduction}

\begin{abstract}
Several million years ago as life forms moved from salt water to fresh water (fish) and sequentially from an amphibious existence to predominantly terrestrial forms, there was evolutionary pressure to evolve a mechanism for salt and water conservation. The mineralocorticoid pathway and its prototype hormone, aldosterone, emerged as vital axes for survival of several land forms, including Homo Sapiens [1-]. Our prehistoric ancestors were hunter gatherers who ate a sodium-restricted potassium-enriched diet consisting of fruits and vegetables [2]. Additionally, early humans faced obligatory sodium loss through sweating in an arid environment and also a likelihood of devastating volume losses due to diarrheal disorders or exsanguinating hemorrhage (due to child birth or injury). Thus, the evolution of pathways for conserving sodium and water and excreting potassium was vital and the reninangiotensin-aldosterone system emerged as the much needed mechanism for survival. In
\end{abstract}

Address for Correspondence: Vanessa Xanthakis, PhD, 801 Massachusetts Avenue, Suite 470, Boston, MA 02118, vanessax@bu.edu. Disclosure No potential conflicts of interest relevant to this article were reported. 
more recent times, in acculturated societies that are salt replete, the same mineralocorticoid pathway serves as double-edged sword with a propensity for elevating blood pressure and contributing to the burden of hypertension [2,3]. In this review, we summarize the molecular mechanisms that underlie aldosterone action and describe the multiple renal and cardiovascular effects of the mineralocorticoid pathway. We review the epidemiology of aldosterone and the aldosterone to renin ratio (ARR) in the community and their likely contribution to the population burden of high blood pressure. We critique recent studies that demonstrate a higher than previously believed prevalence of primary hyperaldosteronism among hypertensive patients, note current recommendations for the diagnosis of the condition, and underscore the use of aldosterone antagonists for treating resistant hypertension.

\section{Aldosterone, Mineralocorticoid Receptor, and Blood Pressure: Physiology and Regulation}

Aldosterone is produced by the adrenal zona glomerulosa cells in response to predominantly three sets of stimuli: local and systemic angiotensin II, circulating adrenocorticotrophic hormone, and serum potassium levels [4-]. All these three stimuli can have both acute and chronic effects on aldosterone synthesis and release. Aldosterone synthesis itself involves an acute phase regulated by a protein called StAR (steroidogenic acute regulatory protein) that is needed for delivery of cholesterol within the zona glomerulosa cells to the inner mitochondrial membrane; the stimuli noted above increase expression and phosphorylation of StAR [4]. There is also a chronic phase of aldosterone secretion in the mitochondria that involves several steroidogenic enzymes of which aldosterone synthase (CYP11B2) is the rate limiting step [4].

Once secreted, aldosterone binds to the mineralocorticoid receptors (MR) in the epithelial cells in the distal convoluted tubule and collecting duct of the nephrons $[4,5 m, 6 m]$. By upregulating the basolateral sodium-potassium exchange pump, the epithelial sodium channels $(\mathrm{ENaC})$, and the outer medullary renal potassium channels, aldosterone promotes sodium and water reabsorption and secretion of potassium into the tubular lumen $[4,6 \mathbf{m}]$.

Aldosterone mediates these effects both by genomic and nongenomic effects. The genomic mechanisms increase the transcription of genes regulating these channels and the exchange pump. The nongenomic effects are mediated either by intracellular receptors ( $\mathrm{G}$ protein coupled receptor 30) or via non-receptor second messenger pathways that can alter intracellular ionic concentrations (including the sodium-hydrogen exchanger) [5m].

Recent studies have demonstrated that the physiological effects of aldosterone are not limited to the MR on the renal epithelial cells $[5 m, 6 m]$. Rather, MR are observed on several non-epithelial cells including cardiomyocytes, cardiac fibroblasts and vascular smooth muscle cells. Aldosterone elicits a fibrotic response in the myocardium, and a profibrotic, hypertrophic and inflammatory response in the vasculature, where it also reduces nitric oxide bioactivity thereby promoting endothelial dysfunction [5m]. Aldosterone influences endothelial cell function adversely by causing the cells to swell and become stiff due to its effect on the antioxidant enzyme glucose-6-phosphate dehydrogenase and epidermal growth factor, by activating phospholipase $\mathrm{C}$, and also by inhibiting the homing of endothelial progenitor cells (see reference 5 for comprehensive review of mechanisms by which aldosterone affects vascular structure and function) [5m]. Thus, aldosterone not only controls salt and water homeostasis but it also influences vascular and cardiac remodeling because of its powerful vasoconstrictor effect on blood vessels, by promoting a profibrotic milieu in the myocardium and blood vessels, and by increasing oxidative stress and inflammation in several of these tissues. Therefore, aldosterone contributes to target organ damage involving the heart, kidneys and the brain, independent of its sodium retaining and 
blood pressure elevating effects. Aldosterone also has adverse metabolic sequelae; the prevalence of the metabolic syndrome is reported to be higher in patients with primary hyperaldosteronism compared to patients with primary hypertension with a similar magnitude of blood pressure elevation [7]. Excess obesity has also been associated with release of select adipokines that are potent stimulators of aldosterone secretion [8]. When evaluating the systemic and cardiovascular effects of aldosterone, it is critical to take circulating renin levels into account because aldosterone and renin can synergistically contribute to the development of cardiovascular disease, including hypertension (see below).

Classically, MR-associated hypertension occurs in the context of higher than normal aldosterone levels [6-1]. In this instance, plasma renin levels are typically low, resulting in an elevated ARR. Classic examples include primary hyperaldosteronism (see below), individuals with higher ARR without primary hyperaldosteronism, patients with 'aldosterone escape' in response to treatment with angiotensin-converting enzyme inhibitor (ACE-I) or angiotensin II receptor blocker (ARB), and people with obstructive sleep apnea [6-1]. Although not the focus of the current review (see reference 6 for a state-of-the-art review of the mechanisms underlying MR-associated hypertension), it is important to note that MR activation may occur without higher aldosterone levels (presumably due to binding of other factors to MR, such as Rac-1) in the setting of obesity, diabetes, chronic kidney disease or polycystic ovary syndrome resulting in salt-sensitive hypertension [6-[]. Thus, increased MR gene transcription may occur in stroke, diabetes and chronic kidney disease [6-1. An increase in sensitivity of the MR to aldosterone (within the normal range) may be seen in conditions associated with oxidative stress. A greater stabilization of the MR transcripts, as seen in .hyperglycemia, may also contribute to greater risk of hypertension [6-1]. Last but not the least, excess salt intake in experimental models results in increased expression of the small GTPase protein Rac-1, which in turn increases MR activity and causes hypertension [6-m].

\section{Epidemiology of Serum Aldosterone, ARR and Risk of HTN and Cardiovascular Disease}

\section{Clinical correlates}

Information regarding the genetic and clinical correlates of aldosterone and the aldosterone/ renin ratio (ARR) in the community is limited, especially among people without hypertension. Kathiresan et al [9] reported a complex association between aldosterone and standard cardiovascular risk factors. In this cross-sectional study, increasing plasma aldosterone concentration was associated with female sex, increased total/HDL cholesterol, and diuretic treatment. On the other hand, a lower aldosterone concentration was directly associated with postmenopausal status (without hormone replacement therapy), a higher urinary sodium, a higher pulse pressure, and prevalent CVD.

Other studies have focused on correlates of ARR in referral samples of individuals with hypertension; in these reports (cited in reference 10) [10], ARR has been directly associated with age, female sex, body mass index, total/high-density lipoprotein cholesterol, obesity, postmenopausal status, blood pressure, hormone replacement therapy, white race, urinary sodium excretion (a marker of dietary salt intake), posture, and time of day. Additionally, increased ARR can be found in patients with hypertension with low renin and normal or even low aldosterone levels. Newton-Cheh et al. reported on the correlates of ARR among non-hypertensive individuals in the community [10]. The correlates of a higher ARR included female sex, age, hypertension status, total/HDL cholesterol ratio, hormone replacement therapy, and beta-blocker use. Additionally, Newton-Cheh et al [10] reported an inverse association between ARR and ACE inhibitor and diuretic use. Both serum 
aldosterone and ARR are heritable phenotypes $[9,10]$, underscoring the importance of additive genetic effects to inter-individual variation in these traits in addition to environmental factors noted above.

\section{Serum Aldosterone and Hypertension Risk}

Vasan et al [3] reported that among 1688 non-hypertensive individuals in the Framingham Heart Study, variation in serum aldosterone levels influenced blood pressure tracking over a 4-year period. The investigators observed that rates of blood pressure progression (by more than $1 \mathrm{JNC}$ VI category) and hypertension incidence rose with increasing serum aldosterone concentrations (Figure 1). In multivariable analyses, each quartile increment in serum aldosterone within the normal range was associated with a $15 \%$ increased risk of blood pressure progression and a $17 \%$ increased risk of hypertension. The top quartile of serum aldosterone was associated with a 1.6-fold risk of blood pressure progression and risk of hypertension relative to the lowest quartile. In a follow-up report [10] that focused on the ARR, the Framingham investigators reported that higher ARR was associated with an increased risk of BP progression and hypertension. The top ARR quartile was associated with a 1.89-fold risk of BP progression and a 1.53-fold risk of hypertension compared to the lowest ARR quartile [10].

\section{Aldosterone and CVD Risk}

The important role of renin-angiotensin-aldosterone system (RAAS) in the development of cardiovascular disease among patients with hypertension has also been underscored by several reports. Patients diagnosed with primary hyperaldosteronism have been reported to experience higher rates of cardiovascular events and end organ damage than those with primary hypertension (with comparable levels of blood pressure elevation) in several reports [11].

More recently, Kisaka et al [12-] compared prognosis of hypertensive patients with a low versus high ARR (defined as below versus above the $50^{\text {th }}$ percentile of the sex-pooled ARR value of 5.5, respectively). The investigators reported that patients in the high ARR group had higher cardiovascular morbidity and overall mortality rates. Specifically, the incidence of CVD events and stroke was higher in individuals in the high ARR group (compared to those in the low ARR group) [12-]. Notably, those with a higher ARR had a 2.7-fold larger incidence of stroke compared to those in the low ARR group [12-]. The addition of ARR in a predictive model for CVD that included the Framingham Risk Score resulted in an increase in model discrimination as quantified by the $\mathrm{C}$ statistic [12-]. Thus, this report [12-] emphasizes that ARR is a key cardiovascular risk factor.

\section{Primary hyperaldosteronism in hypertensive patients}

It has been recognized for several decades (since the initial report by Conn in 1955) that primary hyperaldosteronism is an important cause of secondary hypertension, being typically characterized by high (or occasionally normal) aldosterone levels, suppressed renin levels, and an elevated ARR. The underlying causes of primary hyperaldosteronism include bilateral adrenal hyperplasia (in about $60 \%$ of cases), an aldosterone secreting adrenal adenoma (a cause amenable to surgical treatment), and rarer forms such as glucocorticoid remediable aldosteronism (GRA) and familial hyperaldosteronism Type I. A discussion of the latter forms is beyond the scope of the present review; these entities are reviewed in detail in references 13-15 [5, 13-15]. It is critical to consider the possibility of hyperaldosteronism in a patient with a high pre-test probability of secondary hypertension because [16--]: hypertensive patients with hyperaldosteronism have worse cardiovascular morbidity relative to those with essential hypertension, with rates of stroke and non-fatal 
myocardial infarction being 4-6 fold higher in the former compared to the latter [11]; the former may be curable on occasion surgically (when due to an adenoma), thereby avoiding lifelong antihypertensive treatment; and it may influence the choice of antihypertensive treatment because patients with hyperaldosteronism respond better to MR blockers such as spironolactone.

Whereas there is little dispute that hyperaldosteronism is an important etiologic factor to be considered in patients with a high likelihood of secondary hypertension, the prevalence estimates among hypertensive patients has varied considerably in the literature. Formerly it was widely believed that primary hyperaldosteronism was quite infrequent among patients with hypertension, constituting less than $2 \%$ of all cases (with a range of $0.5-2 \%$ ). Recent estimates have been considerably higher, in part because of the recognition that hypokalemia is not always a concomitant of hyperaldosteronism, and also greater awareness and use of the ARR for the diagnosis of the condition. More recent estimates suggest that the prevalence of primary hyperaldosteronism may vary based on the sampling frame of hypertensive patients and their severity of hypertension [17], and also the criteria used for the diagnosis of the condition. Thus, a recent meta-analysis [17] estimated that the prevalence of hyperaldosteronism is $4.3 \%$ among primary care patients with hypertension ( 8 studies), but higher among referred patients with hypertension in clinical centers (estimated at $9 \%$, based on 12 studies), and even higher among patients with refractory hypertension (in whom prevalence may be as high as $15-20 \%$ ) [18- $\mathbf{m}$.

As noted above, the prevalence of primary hyperaldosteronism also varies based on the specific biochemical criteria used to diagnose the condition. A high ARR has been widely used to detect identifiable secondary causes of hypertension, due to the fact that primary hyperaldosteronism has been known to be one of its most common causes. Yet, sole reliance on the ARR typically overestimates the prevalence of hyperaldosteronism because of various factors that can influence the ratio and impact its sensitivity and specificity for the diagnosis of the condition $[16,19]$. Using data from the Study of Health in Pomerania (SHIP) and the Cooperative Health Research in the Region of Augsburg (KORA) studies, Hannemann et al [20-m] reported the prevalence of primary hyperaldosteronism among patients with hypertension, according to the following three definitions: 1) increased ARR; 2) increased ARR and decreased plasma renin concentrations (PRC); and 3) increased ARR with increased serum aldosterone and decreased PRC. The prevalence of primary hyperaldosteronism using the aforementioned definitions are 5.9, 2.6, and $0.2 \%$ among patients with hypertension who are not on antihypertensive medications, and 8.5, 4.9, and $0.5 \%$ among patients with hypertension who are on antihypertensive medications, for definitions 1 ), 2), and 3), respectively [20-1. Thus, if one uses very stringent criteria for the diagnosis of primary hyperaldosteronism (all 3 conditions, i.e., increased ARR, elevated aldosterone, and decreased PRC), the prevalence is much lower than that using less strict criteria.

Recent guidelines from the Endocrine society [21] emphasize that in addition to estimation of the ARR under standardized conditions, one of 4 confirmatory tests (oral sodium loading, saline infusion, fludrocortisone suppression, and the captopril challenge test) be used to establish a diagnosis of primary hyperaldosteronism, the choice of the confirmatory test in part being determined by local expertise, costs, and laboratory practices [21]. Reference 21 reviews the approach to the diagnosis of primary hyperaldosteronism in detail [21].

\section{Aldosterone, natriuretic peptides, and salt sensitivity}

Increased dietary salt intake in the context of greater "salt sensitivity" has been associated with higher incidence of cardiovascular disease and also cardiovascular target organ 
damage. More specifically, increased dietary salt intake imposed on a background of higher circulating aldosterone levels, and lower blood N-terminal proatrial natriuretic peptide (NTANP) is associated with "salt sensitivity" [22-]. Lieb et al [22-] investigated this premise and reported a higher increase in blood pressure (both systolic and diastolic) among nonhypertensive individuals who were "salt sensitive" (i.e., having a serum aldosterone > sexspecific median and plasma NT-ANP $\leq$ sex-specific median) when placed on a higher sodium diet (i.e., > sex-specific median) as compared to others, which suggests that the relative balance of aldosterone and NT-ANP may serve as a marker of salt sensitivity in the community [22-].

\section{Aldosterone and Resistant HTN}

Several recent investigations have elegantly demonstrated the efficacy of spironolactone treatment in individuals with high blood pressure refractory to conventional treatment regimen $[18 \boldsymbol{m}, 23 \boldsymbol{m}]$. Of note, spironolactone is effective in lowering blood pressure in these patients regardless of serum aldosterone levels. This topic has been extensively reviewed elsewhere (see reference 18) [18m]. Potential explanations include the possibility that aldosterone levels in the tissues may be pathogenic even though circulating levels are in a 'normal' range, and also the possibility that factors besides aldosterone may activate the MR and raise BP (and spironolactone antagonizes the MR) [18m].

\section{Conclusions}

Aldosterone is a fundamental regulator of blood volume and blood pressure. Aldosterone also influences cardiac and vascular remodeling due to its profibrotic, hypertrophic and prooxidant effects. Recent investigations have demonstrated that within the normal range both a higher aldosterone level and a higher ARR serve as markers of future hypertension risk. Some recent investigations have underscored a higher than previously thought prevalence of primary hyperaldosteronism among hypertensive patients, especially in those with more severe or resistant hypertension and in patients seen in hypertension clinics. Recent guidelines from the Endocrine society [21] have proposed a systematic approach towards the diagnosis of the condition using screening ARR and follow-up confirmatory tests. It is likely that the mineralocorticoid pathway exhibits "cross-talk" with other biochemical pathways and the balance of natriuretic and sodium retaining pathways may determine salt sensitivity and propensity for developing high blood pressure.

\section{Acknowledgments}

This work was supported in part by NIH/NHLBI contract NO1-HC-25915 (R.S. Vasan).

\section{References}

Papers of particular interest, published recently, have been highlighted as:

- Of importance

•- Of major importance

1. Milliez P, Girerd X, Plouin PF, et al. Emergence and evolution of the renin-angiotensin-aldosterone system. J Mol Med (Berl). 2012; 90:495-508. This report presents a phylogenetic analysis of the evolution of the renin-aniotensin-aldosterone systems and notes that about 400 million years ago the pathway evolved in primitive chordates as the need for salt conservation and blood pressure maintenance arose. [PubMed: 22527880]

2. Harris P. Evolution and the cardiac patient. Cardiovascular Research. 1983; 17:437-445. [PubMed: 6352032] 
3. Vasan RS, Evans JC, Larson MG, et al. Serum aldosterone and the incidence of hypertension in nonhypertensive persons. N Engl J Med. 2004; 351:33-41. [PubMed: 15229305]

4". Hattangady NG, Olala LO, Bollag WB, Rainey WE. Acute and chronic regulation of aldosterone production. Molecular and Cellular Endocrinology. 2012; 350:151-162. This report discusses the molecular mechanisms underlying the acute and chronic phases of aldosterone secretion in response to various physiological stimuli. [PubMed: 21839803]

5-m. Briet M, Schiffrin EL. Vascular Actions of Aldosterone. J Vasc Res. 2012; 50:89-99. This is a comprehensive review of the multiple molecular mechanisms by which aldosterone impairs endothelial function. [PubMed: 23172373]

6m. Shibata H, Itoh H. Mineralocorticoid Receptor-Associated Hypertension and Its Organ Damage: Clinical Relevance for Resistant Hypertension. Am J Hypertens. 2012; 25:514-523. This is a state-of-the-art review that discusses the pathogensis of the two categories of mineralocorticoidassociated hypertension (with elevated and normal aldosterone levels) and disease mechanisms underlying this state. [PubMed: 22258336]

7. Fallo F, Veglio F, Bertello C, et al. Prevalence and Characteristics of the Metabolic Syndrome in Primary Aldosteronism. Journal of Clinical Endocrinology \& Metabolism. 2006; 91:454-459. [PubMed: 16291704]

8. Ehrhart-Bornstein M, Lamounier-Zepter V, Schraven A, et al. Human adipocytes secrete mineralocorticoid-releasing factors. Proceedings of the National Academy of Sciences. 2003; 100:14211-14216.

9. Kathiresan S, Larson MG, Benjamin EJ, et al. Clinical and genetic correlates of serum aldosterone in the community: the Framingham Heart Study. Am J Hypertens. 2005; 18:657-665. [PubMed: 15882548]

10. Newton-Cheh C, Guo CY, Gona P, et al. Clinical and genetic correlates of aldosterone-to-renin ratio and relations to blood pressure in a community sample. Hypertension. 2007; 49:846-856. [PubMed: 17296870]

11. Milliez P, Girerd X, Plouin PF, et al. Evidence for an increased rate of cardiovascular events in patients with primary aldosteronism. J Am Coll Cardiol. 2005; 45:1243-1248. [PubMed: 15837256]

12. Kisaka T, Ozono R, Ishida T, et al. Association of elevated plasma aldosterone-to-renin ratio with future cardiovascular events in patients with essential hypertension. J Hypertens. 2012; 30:23222330. Among Japanese patienst with hypertension followed up for up to 9 years, those with higher ARR (above the median) had a 2.7-fold greater risk of stroke, and addition of ARR to the Framingham risk score increased model performance as evaluated by discrimination index (the C-statistic). [PubMed: 23107914]

13. Stowasser M, Huggard PR, Rossetti TR, et al. Biochemical Evidence of Aldosterone Overproduction and Abnormal Regulation in Normotensive Individuals with Familial Hyperaldosteronism Type I. J Clin Endocrinol Metab. 1999; 84:4031-4036. [PubMed: 10566645]

14. Stowasser M, Bachmann AW, Huggard PR, et al. Severity of Hypertension in Familial Hyperaldosteronism Type I: Relationship to Gender and Degree of Biochemical Disturbance. J Clin Endocrinol Metab. 2000; 85:2160-2166. [PubMed: 10852446]

15. White PC. Inherited Forms of Mineralocorticoid Hypertension. Hypertension. 1996; 28:927-936. [PubMed: 8952579]

16m. Kaplan NM. Primary aldosteronism: evidence against a second epidemic. J Hypertens. 2012; 30:1899-1902. This review highlights the limitations of the ARR as a sole diagnostic tool for making a diagosis of hyperaldosteronism and discusses how referral bias inflates prevalence estimates of the condition among hypertensive patients in different settings (primary care clinic vs. hypertension clinic vs resistant hypertension). [PubMed: 22929608]

17. Jansen PM, Boomsma F, van den Meiracker AH. Aldosterone-to-renin ratio as a screening test for primary aldosteronism--the Dutch ARRAT Study. Neth J Med. 2008; 66:220-228. [PubMed: 18490805]

18m. Clark D III, Ahmed MI, Calhoun DA. Resistant hypertension and aldosterone: an update. Can J Cardiol. 2012; 28:318-325. This report highlights the high prevalence of primary hyperaldosteronism in patients with resistant hypertension, discusses the good response to 
spironolactone treatment in these patients and highlights the assocaition of hyperaldosteronism with obstructive sleep apnea. [PubMed: 22521297]

19. Kaplan NM. Cautions over the current epidemic of primary aldosteronism. The Lancet. 2001; 357:953-954.

20m. Hannemann A, Bidlingmaier M, Friedrich N, et al. Screening for primary aldosteronism in hypertensive subjects: results from two German epidemiological studies. Eur J Endocrinol. 2012; 167:7-15. This report elucidates how use of 3 different sets of diagnostic criteria (using a combination of high ARR, high serum aldosterone and reduced plasma renin concentrations) can result in widely varying prevalence estimates for primaty hyperaldosteronism in hyperetnsive patients in the community. [PubMed: 22495491]

21. Funder JW, Carey RM, Fardella C, et al. Case Detection, Diagnosis, and Treatment of Patients with Primary Aldosteronism: An Endocrine Society Clinical Practice Guideline. Journal of Clinical Endocrinology \& Metabolism. 2008; 93:3266-3281. [PubMed: 18552288]

22-. Lieb W, Pencina MJ, Jacques PF, et al. Higher aldosterone and lower N-terminal proatrial natriuretic peptide as biomarkers of salt sensitivity in the community. Eur J Cardiovase Prev Rehabil. 2011; 18:664-673. This investigation demonstrates how the combination of higher salt intake, higher serum aldosterone and lower N-terminal proatrial natriuretic peptide concentratiosn can translate into a greater risk of developing hyperetnsion prospectively among community-dwelling middle-aged adults. [PubMed: 21450637]

23m. Clark D III, Guichard JL, Calhoun DA, Ahmed MI. Recent advancements in the treatment of resistant hypertension. Postgrad Med. 2012; 124:67-73. This review discusses the importance of aldosterone excess and responsiveness to spironolactone treatment in patients with resistant hypertension. [PubMed: 22314116] 


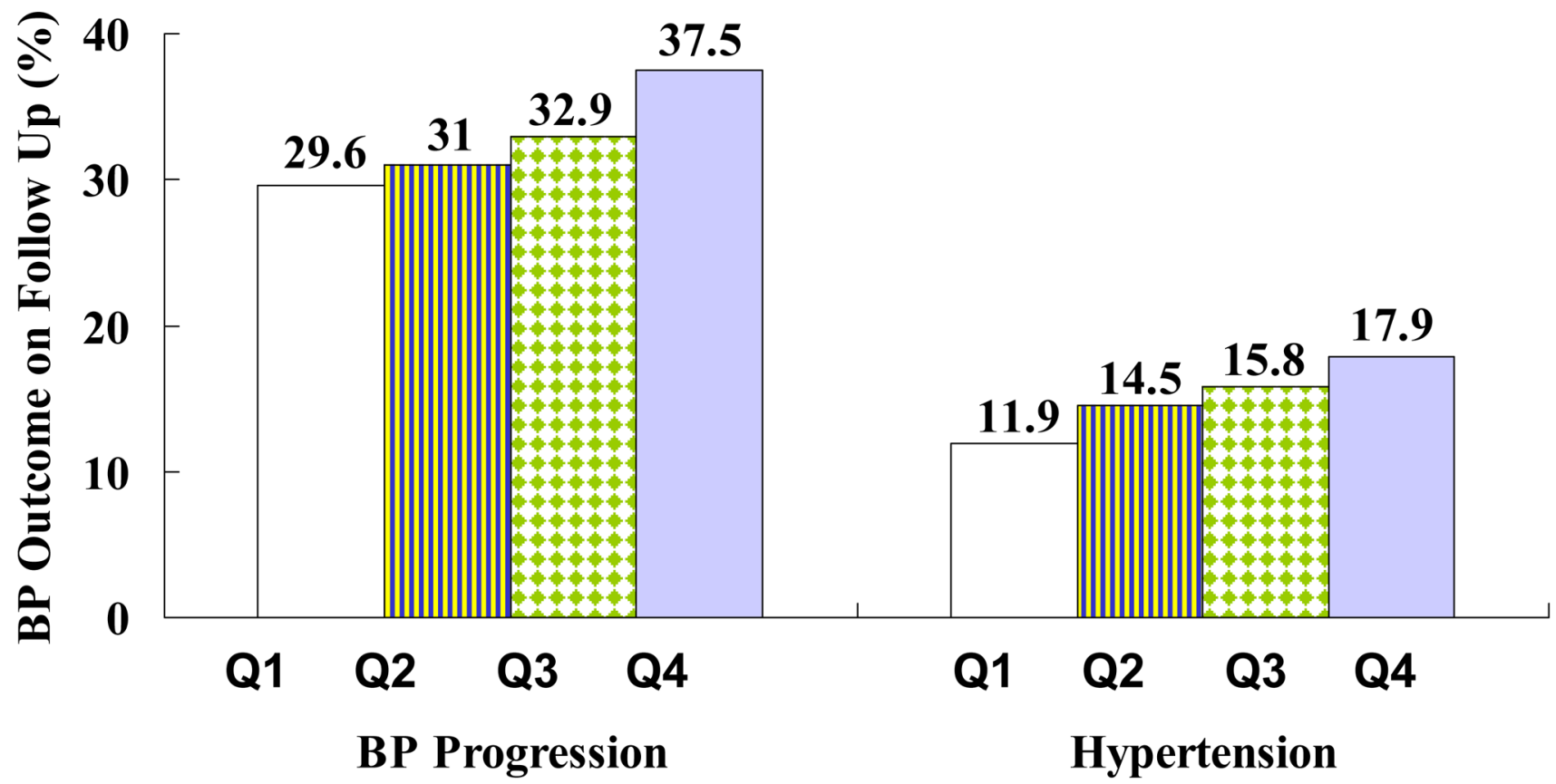

Figure 1.

Age- and sex-adjusted 4-year rates of blood pressure progression (increase of blood pressure by $\geq 1$ stage) and incidence of hypertension according to serum aldosterone quartiles. From N Engl J Med, Vasan RS, Evans JC, Larson MG, Wilson PW, Meigs JB, Rifai N, Benjamin EJ, Levy D, "Serum aldosterone and the incidence of hypertension in nonhypertensive persons," 351:33-41. Copyright @ (2004) Massachusetts Medical Society. Reprinted with permission from reference 3 . 